\title{
EFEITO DOS SUBSTRATOS E TEMPERATURAS NA GERMINAÇÃO DE SEMENTES DE Cnidosculus phyllacanthus Pax \& K. Hoffm. (FAVELEIRA) ${ }^{1}$
}

\author{
LÍGIA MARIA DE MEDEIROS SILVA²; IVOR BERGEMANN DE AGUIAR ${ }^{3}$
}

\begin{abstract}
RESUMO - Este trabalho foi conduzido com o objetivo estudar diferentes substratos e temperaturas para germinação de sementes de faveleira. Foram testadas as temperaturas constantes de $25^{\circ}$ e $30^{\circ} \mathrm{C}$ e alternadas com amplitudes de $5^{\circ} \mathrm{C}\left(30-35^{\circ} \mathrm{C}\right), 10^{\circ} \mathrm{C}\left(20-30^{\circ}\right.$ e $25-$ $\left.35^{\circ} \mathrm{C}\right)$ e $15^{\circ} \mathrm{C}\left(20-35^{\circ} \mathrm{C}\right)$, e fotoperíodo de oito horas para a temperatura mais elevada e dezesseis horas para a temperatura mais baixa. Os substratos papel de filtro, areia, vermiculita e papel germitest, foram umedecidos com solução de nistatina $0,2 \%$. Para o teste de germinação em laboratório para sementes de faveleira pode-se recomendar os substratos areia, vermiculita, papel germitest e papel filtro combinado com temperaturas alternadas de $20^{\circ} \mathrm{C}-30^{\circ} \mathrm{C}$. Para o teste de vigor, velocidade de germinação de sementes de faveleira, pode-se recomendar o substrato papel filtro combinado com as temperaturas alternadas de $20^{\circ} \mathrm{C}-30^{\circ} \mathrm{C}$.
\end{abstract}

Termos para indexação: Espécie florestal, análise de sementes, qualidade fisiológica.

\section{EFFECT OF SUBSTRATES AND TEMPERATURES ON GERMINATION OF Cnidosculus phyllacanthus Pax \& K. Hoffm SEEDS}

\begin{abstract}
This study had as objective to investigate different conditions of substrate and temperature regime of Cnidosculus phyllacanthus. Two experiments were carried out and the germination tests were conducted with an eight-hour photoperiod. In the first experiment four substrates were tested (on filter paper, on sand and on vermiculite, as well as rolled paper) and two temperature regimes: constant $\left(25^{\circ}\right.$ and $\left.30^{\circ} \mathrm{C}\right)$ and alternating $\left(20-30^{\circ}\right.$ and $\left.25-35^{\circ} \mathrm{C}\right)$. The results showed that: the better germination behaviour was found under the regime of alternating temperature $\left(20-30^{\circ} \mathrm{C}\right)$, seed germination capacity was not affected by the tested substrates; and vermiculite showed advantages of practical aspects for use as substrate. In the second experiment alternating temperatures with amplitudes of $5^{\circ} \mathrm{C}(30$ $\left.35^{\circ} \mathrm{C}\right), 10^{\circ} \mathrm{C} 20-30^{\circ}$ and $\left.25-35^{\circ} \mathrm{C}\right)$ and $15^{\circ} \mathrm{C}\left(20-35^{\circ} \mathrm{C}\right)$ were tested. The results showed that amplitudes of $10^{\circ}$ and $15^{\circ} \mathrm{C}$, when the lower temperature was $20^{\circ} \mathrm{C}\left(20-30^{\circ}\right.$ and $\left.20-35^{\circ} \mathrm{C}\right)$, favoured seed germination.
\end{abstract}

Index terms: Forest specie, seed analysis, physiological quality.

\section{INTRODUÇÃO}

Um dos meios utilizados para se determinar o nível de qualidade das sementes é o teste padrão de germinação, o qual é realizado sob condições de temperatura e substrato ideais para cada espécie (Gomes e Bruno, 1992).

As sementes de diferentes espécies apresentam comportamentos variáveis para a temperatura, o que pode fornecer informações de interesse biológico e ecológico (Labouriau, 1983). Dentro da faixa de temperatura em que

\footnotetext{
${ }^{1}$ Submetido em 21/07/2003. Aceito para publicação em 16/01/2004

${ }^{2}$ Bolsista FAPESP, doutoranda em Agronomia (Produção e Tecnologia de Sementes), FCAV/UNESP, Jaboticabal,SP. E-mail: ligiamms@hotmail.com

${ }^{3}$ Bolsista do CNPq, Prof ${ }^{\circ}$ Voluntário do Departamento de Produção vegetal/FCAV/UNESP, Jaboticabal,SP. E-mail: ivor@netsite.com.br
}

as sementes de uma espécie germinam, há uma temperatura ótima, denominada como aquela em que ocorre o máximo de germinação em menor intervalo de tempo. Temperaturas mínima e máxima são aquelas em que a germinação é zero (Mayer e Poljakoff-Mayber, 1989; Borges \& Rena, 1993).

Segundo Copeland e McDonald (1995), determinadas espécies apresentam melhor comportamento germinativo quando submetidas à alternância de temperatura. Essa alternância de temperatura corresponde às flutuações naturais encontradas no ambiente de clareira. Existem espécies que a germinação de suas sementes é favorecida quando submetidas à temperatura constante (Lima et al., 1997); outras exigem alternância de temperatura (Salomão et al., 1995) e existem ainda espécies que germinam indiferentemente em temperaturas constantes ou alternadas (Albuquerque et al., 1998). 
O substrato utilizado nos testes de germinação também apresenta grande influência na germinação, pois fatores como aeração, estrutura, capacidade de retenção de água, grau de infestação de patógenos, entre outros, podem variar de um substrato para outro, favorecendo ou prejudicando a germinação das sementes (Popinigis, 1985). $\mathrm{Na}$ escolha do material para substrato, deve ser levado em consideração o tamanho da semente, sua exigência com relação à umidade, sensibilidade ou não à luz, a facilidade que este oferece para o desenvolvimento e a avaliação das plântulas (Figliolia et al., 1993).

Pereira \& Andrade (1994) recomendaram o uso de temperatura alternada na faixa de $20-30^{\circ} \mathrm{C}$ ou $15-35^{\circ} \mathrm{C}$, sob vermiculita, papel de filtro ou papel toalha para o teste de germinação de sementes de Psidium guajava (goiabeira). Albuquerque et al. (1998) conduziram estudos com sementes de Colubrina glandulosa (saraguagi) e recomendaram as temperaturas de $25^{\circ}, 30^{\circ}$ e $20-30^{\circ} \mathrm{C}$ e os substratos sobre e entre vermiculita, areia e papel de filtro para o teste de germinação das sementes. Medeiros e Zanon (1998) recomendaram a utilização do substrato papel de filtro e a temperatura de $30^{\circ} \mathrm{C}$ para a germinação de sementes de Sebastiania commersoniana (branquilho) e papel de filtro e areia, na temperatura de $25^{\circ} \mathrm{C}$, para Podocarpus lambertii (pinheiro-bravo). Fowler e Carpanezzi (1998) recomendaram para o teste de germinação de sementes de Mimosa bimucronata os substratos papel toalha, papel mata-borrão, areia ou vermiculita, na temperatura de $25^{\circ} \mathrm{C}$.

Dotada de grande resistência à seca, a faveleira é uma planta rústica e de rápido crescimento, podendo ser usada para composição de reflorestamento destinados à recuperação de áreas degradadas. É uma planta seletiva higrófita, pioneira, exclusiva das matas xerófitas (caatinga) do nordeste brasileiro, onde ocorre com elevada freqüência e irregular dispersão (Lorenzi, 1998).

O presente trabalho foi conduzido com o objetivo de definir diferentes substratos etemperaturas mais adequadas para a germinação de sementes de faveleira em laboratório.

\section{MATERIAL E MÉTODOS}

Foram conduzidos dois experimentos, um para testar o efeito dos substratos e temperaturas na germinação das sementes, e outro para testar os efeitos de diferentes temperaturas alternadas na germinação das sementes de faveleira.

Efeito dos substratos combinados com temperaturas constantes e alternadas - Os substratos testados foram papel de filtro, areia, vermiculita e papel germitest, combinados com as temperaturas constantes de $25^{\circ}$ e $30^{\circ} \mathrm{C}$ e alternadas de $20-30^{\circ}$ e $25-35^{\circ} \mathrm{C}$. As avaliações foram feitas até o vigésimo-quinto dia após a instalação do experimento.
Temperaturas alternadas - Considerando que a alternância de temperaturas observada no experimento um favoreceu a germinação das sementes, foram testadas temperaturas alternadas nas amplitudes de $10^{\circ} \mathrm{C}\left(20-30^{\circ} \mathrm{C}\right.$ e $\left.25-35^{\circ} \mathrm{C}\right), 5^{\circ} \mathrm{C}\left(30-35^{\circ} \mathrm{C}\right)$ e $15^{\circ}\left(20-35^{\circ} \mathrm{C}\right)$.

Foi utilizado o substrato sobre vermiculita por ter apresentado melhor desempenho dentre os testados, e mantendo-se úmido por mais tempo, dispensando o reumedecimento diário durante a execução do experimento.

Os testes de germinação tiveram a duração de 18 dias. Os cálculos de freqüência relativa de germinação foram realizados conforme fórmulas descritas por Labouriau \& Agudo (1987).

Procedimentos - Os experimentos foram conduzidos no Laboratório de Análise de Sementes do Departamento de Produção Vegetal da Faculdade de Ciências Agrárias e Veterinárias da Universidade Estadual PaulistaJaboticabal,SP. As sementes de faveleira foram colhidas no município de Patos-PB, em agosto de 1999. Em seguida foram secas à sombra, acondicionadas em saco de polietileno e armazenadas em câmara fria $\left(10^{\circ} \mathrm{C}\right.$ e $75 \%$ UR) até a instalação do experimento, em janeiro de 2000. Para diminuir a ação de patógenos durante a germinação, as sementes foram desinfestadas com água sanitária a $4 \%$ durante 10 minutos, em seguida lavadas com água destilada. Para acelerar e uniformizar a germinação, as sementes foram mantidas em água a $30^{\circ} \mathrm{C}$ durante quatro horas (Silva et. al, 1999).

Os testes de germinação foram conduzidos em germinadores de câmara, tipo B.O.D. Para as temperaturas constantes foi utilizado o fotoperíodo de oito horas. Para as temperaturas alternadas o fotoperíodo coincidiu com a exposição das sementes às temperaturas mais altas. Todos os tratamentos foram conduzidos com cinco repetições de 20 sementes.

Os substratos foram umedecidos com solução de nistatina $0,2 \%$, sendo os substratos papel de filtro, areia e vermiculita colocados em caixa plástica tipo gerbox. $\mathrm{O}$ substrato rolo de papel foi umedecido na proporção de 2,5 vezes o peso do papel.

As contagens das sementes germinadas foram feitas diariamente até o vigésimo-quinto dia após a instalação do experimento. Foram consideradas germinadas as sementes que apresentaram raiz principal igual ou maior que um centímetro. Os dois experimentos foram instalados em delineamento inteiramente casualizado, e o segundo em esquema fatorial $4 \times 4$.

Os cálculos de porcentagem e velocidade de germinação foram realizados utilizando-se as fórmulas descritas por Labouriau \& Agudo (1987). Os resultados de porcentagem foram transformados em arcsen $\sqrt{\%}$ para fins de análise estatística. A análise estatística foi feita com o programa ESTAT desenvolvido pela FCAV/UNESP, Jaboticabal,SP. Para comparação entre as médias foi utilizado o teste de Tukey a $5 \%$ de probabilidade. 


\section{RESULTADOS E DISCUSSÃO}

Os dados referentes à porcentagem e velocidade de germinação das sementes de faveleira em diferentes substratos e temperaturas, estão apresentados nas Tabelas 1 e 2 .

Verificou-se interação significativa entre substrato e temperatura para germinação (Tabela 1) e velocidade de germinação (Tabela 2).

Para o substrato papel, as temperaturas alternadas de $20^{\circ} \mathrm{C}-30^{\circ} \mathrm{C}$ proporcionaram os melhores resultados de germinação e velocidade de germinação. Nas temperaturas constantes de $25^{\circ}$ e $30^{\circ} \mathrm{C}$, houve significativa redução na germinação e na velocidade de germinação em comparação com as temperaturas alternadas. Salomão et al. (1995) também verificaram que as sementes de Dalbergia nigra apresentaram melhor germinação na temperatura alternada de $20^{\circ} \mathrm{C}-30^{\circ} \mathrm{C}$ em substrato papel filtro.

Observa-se, de modo geral, que as temperaturas alternadas de $20^{\circ} \mathrm{C}-30^{\circ} \mathrm{C}$ foi o melhor tratamento para a germinação das sementes. Nessa temperatura não houve diferença entre os substratos testados, contudo a maior velocidade de germinação foi obtida no substrato papel filtro. Segundo VázquezYanes e Orozco Segovia (1984), para espécies cujas sementes estão adaptadas a responder a flutuações térmicas, existem mecanismos enzimáticos que funcionam em diferentes temperaturas. Assim, a germinação ocorre adequadamente apenas quando houver variação térmica durante $\mathrm{o}$ processo catalizado por essas enzimas. Cícero (1986) explica que a alternância de temperatura age sobre o tegumento das sementes, tornando-o mais permeável à água e ao oxigênio e parece agir também sobre o equilíbrio entre as substâncias promotoras e inibidoras da germinação. Oliveira et al. (1989) recomendam que sejam incluídas temperaturas alternadas para as pesquisas relacionadas à metodologia de análise de germinação de sementes florestais, uma vez que elas simulam as flutuações de temperatura que ocorrem próximo ao solo, sob condições naturais.

No substrato papel de filtro observou-se menor capacidade de retenção de água, sendo necessário reumedecê-lo durante o teste. Brasil (1992) recomenda evitar, sempre que possível, a operação de reumedecimento do substrato, após a semeadura, uma vez que pode causar variações adicionais nos resultados, além do inconveniente do reumedecimento quase que diário, este tipo de substrato propicia o aparecimento de fungos.

O substrato areia, apesar do bom desempenho, apresenta o inconveniente de drenar excessivamente a água, ficando a parte superior ressecada. Além disso, é muito pesado e de

difícil manuseio no gerbox (Figliolia et al., 1993). A vermiculita além de apresentar bons resultados é de fácil manuseio, inorgânica, neutra, leve e com boa capacidade de absorção e retenção de água, razão pela qual vem sendo bastante utilizado para os testes com espécies florestais (Figliolia et al.,1993).

Temperaturas Alternadas - Considerando que a alternância de temperaturas foi $\mathrm{o}$ tratamento cujos resultados foram mais favoráveis à germinação das sementes de faveleira, foram testados os efeitos de outros intervalos de alternância de temperaturas.

Na Figura 1 observa-se que ocorreram diferenças significativas na germinação das sementes entre os intervalos de temperaturas testados. As temperaturas alternadas de $20^{\circ}-30^{\circ} \mathrm{C}$ e $20^{\circ}-35^{\circ} \mathrm{C}$ não diferiram

TABELA 1. Germinação média de sementes de Cnidosculus phyllacanthus submetidas a diferentes substratos e temperaturas.

\begin{tabular}{|c|c|c|c|c|c|}
\hline \multirow{3}{*}{ Substratos } & \multicolumn{5}{|c|}{ Germinação $^{1}(\%)$} \\
\hline & \multicolumn{5}{|c|}{ Temperaturas ${ }^{\circ} \mathrm{C}$} \\
\hline & 25 & 30 & 20-30 & 25-35 & Médias \\
\hline Palel germitest & $25,0 \mathrm{~B} \mathrm{c}$ & $42,0 \mathrm{~A} \mathrm{bc}$ & $88,0 \mathrm{~A} \mathrm{a}$ & $59,0 \mathrm{AB} \mathrm{b}$ & $33,5 \mathrm{~B}$ \\
\hline Areia & $53,0 \mathrm{~A} \mathrm{~b}$ & $67,0 \mathrm{~A}$ ab & $82,0 \mathrm{~A} \mathrm{a}$ & $50,0 \mathrm{~B} \mathrm{~b}$ & $63,0 \mathrm{AB}$ \\
\hline Vermiculita & $35,0 \mathrm{AB} \mathrm{c}$ & $68,0 \mathrm{~A} \mathrm{~b}$ & $87,0 \mathrm{~A} \mathrm{a}$ & 80,0 Aa b & $67,5 \mathrm{~A}$ \\
\hline Papel filtro & $54,0 \mathrm{~A} \mathrm{~b}$ & $52,0 \mathrm{~A} \mathrm{~b}$ & $83,0 \mathrm{~A} \mathrm{a}$ & $57,0 \mathrm{AB} b$ & $61,5 \mathrm{AB}$ \\
\hline Médias & $41,8 \mathrm{c}$ & $57,3 \mathrm{~b}$ & $85,0 \mathrm{a}$ & $54,8 \mathrm{~b}$ & \\
\hline $\begin{array}{l}\mathrm{F}(5 \%) ; \mathrm{Tem} \\
18,02\end{array}$ & 4) $3,43^{\circ}$ & (B) & & & \\
\hline
\end{tabular}

TABELA 2. Valores médios de velocidade de germinação de sementes de Cnidosculus phyllacanthus submetidas a diferentes substratos e temperaturas.

\begin{tabular}{|c|c|c|c|c|c|}
\hline \multirow{3}{*}{ Substratos } & \multicolumn{5}{|c|}{ Germinação $^{1}(\%)$} \\
\hline & \multicolumn{5}{|c|}{ Temperaturas ${ }^{\circ} \mathrm{C}$} \\
\hline & 25 & 30 & $20-30$ & $25-35$ & Médias \\
\hline Palel germitest & $0,07 \mathrm{~B} \mathrm{~b}$ & $0,08 \mathrm{~A} \mathrm{~b}$ & $0,14 \mathrm{C} \mathrm{a}$ & $0,13 \mathrm{AB}$ a & $0,11 \mathrm{~B}$ \\
\hline Areia & $0,15 \mathrm{~A} a b$ & $0,06 \mathrm{~A} \mathrm{c}$ & $0,18 \mathrm{~B}$ a & $0,14 \mathrm{~A} \mathrm{~b}$ & $0,13 \mathrm{~A}$ \\
\hline Vermiculita & $0,16 \mathrm{~A} \mathrm{ab}$ & $0,07 \mathrm{~A} \mathrm{c}$ & $0,18 \mathrm{~B}$ a & $0,13 \mathrm{AB} \mathrm{b}$ & $0,13 \mathrm{~A}$ \\
\hline Papel filtro & $0,16 \mathrm{~A} \mathrm{~b}$ & $0,06 \mathrm{~A} \mathrm{~d}$ & $0,23 \mathrm{~A} a$ & $0,11 \mathrm{~B} \mathrm{c}$ & $0,14 \mathrm{~A}$ \\
\hline Médias & $0,13 \mathrm{~b}$ & $0,07 \mathrm{c}$ & $0,18 \mathrm{a}$ & $0,12 \mathrm{~b}$ & \\
\hline
\end{tabular}


significativamente entre si e apresentaram maior germinação $92 \%$ e $94 \%$ respectivamente. Houve diferenças significativas entre essas temperaturas $25-35^{\circ}$ e $30-35^{\circ} \mathrm{C}$. Santos e Aguiar (2000) observaram que a temperatura alternada de $20-30^{\circ} \mathrm{C}$ proporcionou máxima germinação em menor período de tempo em sementes de Sebastiania commersoniana (branquilho). No entanto, Amaral e Paulilo (1992) verificaram que as temperaturas alternadas de $20-25^{\circ}$ e $25-35^{\circ} \mathrm{C}$ inibiram a germinação de sementes de Miconia ciannamomifolia. Segundo Arnold et al. (1988), a alternância de temperatura é o gatilho para a germinação de sementes de Sorghum halepense (L.).

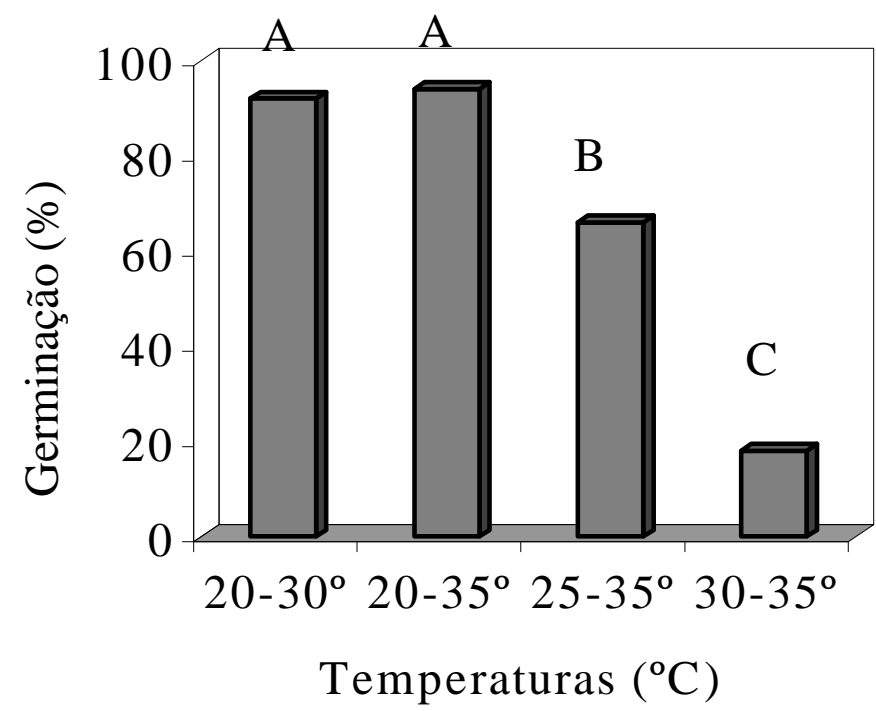

FIGURA 1. Germinação de sementes de Cnidosculus
phyllacanthus submetidas a diferentes
intervalos de alternância de temperaturas.

A menor germinação, $18 \%$, foi observada nas temperaturas alternadas de $30-35^{\circ} \mathrm{C}$. A temperatura de 25 $35^{\circ} \mathrm{C}$ também proporcionou baixa porcentagem de germinação diferindo significativamente das temperaturas alternadas de $20-30^{\circ}$ e $20-35^{\circ} \mathrm{C}$.

Segundo Copeland e McDonald (1995), determinadas espécies apresentam melhor comportamento germinativo quando submetidas à alternância de temperatura. Essa alternância corresponde às flutuações naturais encontradas no ambiente de clareira e parece estar associada com a quebra da dormência. A flutuação de temperatura é um mecanismo que controla eventos de colonização no tempo e no espaço, proporcionando a quebra da dormência em ambiente de clareira, onde as condições são relativamente favoráveis ao estabelecimento de plântulas de algumas espécies de leguminosas Moreno-Casasola et al. (1994). Vázquez-Yanes e Orozco-Segovia (1982) mencionaram que quando uma clareira é aberta, além da mudança na intensidade de luz, ocorre também um aumento na flutuação de temperatura diária na superfície do solo. Durante dias de sol, a temperatura do solo pode subir até $15^{\circ} \mathrm{C}$ acima da temperatura normal do solo da floresta.

A velocidade de germinação foi maior a $20-35^{\circ} \mathrm{C}$ (Figura 2), porém esta temperatura não diferiu significativamente de $20-30^{\circ}$ e $25-35^{\circ} \mathrm{C}$. A menor velocidade de germinação ocorreu na temperatura de 30 $35^{\circ} \mathrm{C}$. A flutuação de $10^{\circ}$ e $15^{\circ} \mathrm{C}$, influenciaram mais a velocidade de germinação do que flutuação de $5^{\circ} \mathrm{C}$.

Segundo Thompson et al. (1977), as flutuações diurnas de temperatura podem iniciar ou acelerar a germinação de sementes de muitas espécies, variando a efetividade do estímulo de acordo com a amplitude de flutuação.

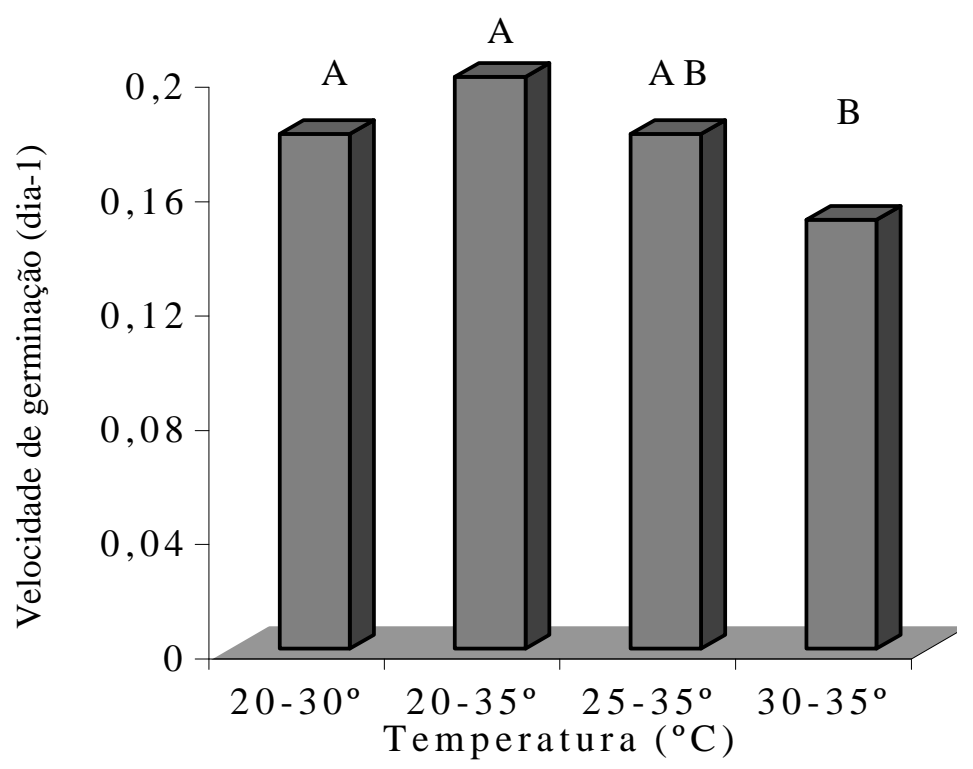

FIGURA.2. Velocidade de sementes de Cnidosculus phyllacanthus submetidas a diferentes intervalos de alternância de temperaturas.

Os polígonos de freqüência relativa de germinação (Figura 3) para as temperaturas de $20-30^{\circ}, 20-35^{\circ}$ e $25-35^{\circ} \mathrm{C}$ apresentaram um pico de germinação, caracterizando germinação mais homogênea. A germinação iniciou no segundo dia para as temperaturas de $20-35^{\circ}$ e $25-35^{\circ} \mathrm{C}$ e no terceiro dia para a temperatura de $20-30^{\circ} \mathrm{C}$.

A temperatura de $30-35^{\circ} \mathrm{C}$ resultou em gráfico polimodal, caracterizando germinação heterogênea, com maior tempo médio de germinação (Figura 3). Nesta temperatura, a germinação teve início no quinto dia após semeadura. 

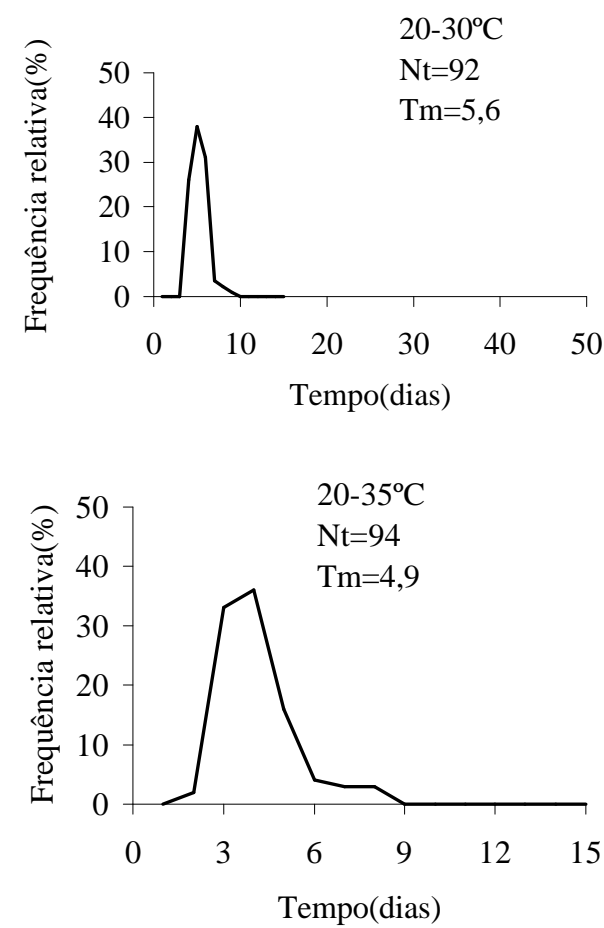
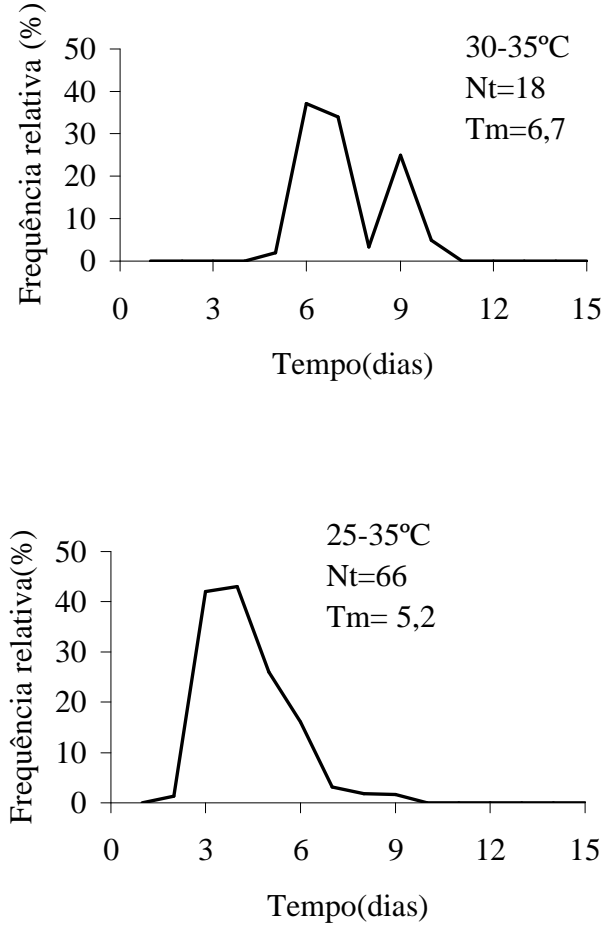

FIGURA 3. Polígonos de freqüência relativa diária da germinação de Cnidosculus phyllacanthus submetidas a diferentes intervalos de alternância de temperaturas $(\mathrm{Nt}=$ número total de sementes germinadas; Tm= tempo médio de germinação).

\section{CONCLUSÕES}

Para o teste de germinação em laboratório para sementes de faveleira pode-se recomendar os substratos areia, vermiculita, papel germitest e papel filtro combinado com temperaturas alternadas de $20^{\circ} \mathrm{C}-30^{\circ} \mathrm{C}$;

Para o teste de vigor, velocidade de germinação de sementes de faveleira, pode-se recomendar o substrato papel filtro combinado com as temperaturas alternadas de $20^{\circ} \mathrm{C}-30^{\circ} \mathrm{C}$.

\section{REFERÊNCIAS}

ABUQUERQUE, M.C.F.; RODRIGUES, T.J.D.; MINOHARA, L.; TEBALDI, N.D.; SILVA, L.M.M. Influência da temperatura e do substrato na germinação de sementes de saguaragi (Colubrina glandulosa Perk)Rhamanaceae. Revista Brasileira de Sementes, Brasília, v. 20, n.2, p.346-349, 1998.

AMARAL, L.I.V.; PAULILO, M.T.S. Efeito da luz, temperatura, regulador de crescimento e nitrato de potássio na germinação de Miconia ciannamomifolia (DC). Insula, Florianópolis, n.21, p.59-86, 1992.
ARNOLD, R.L.B.; GHERSA, C.M.; SANCHEZ, R.A.; FERNANDEZ, A.E.G. The role of fluctuating temperatures in the germination and establishment of Sorghum halepense (L) Pers.: regulation of germination under leaf canpies. Funct. Ecology, v.2, p.311-318, 1988.

BORGES, E.E.L.; RENA, A.B. Germinação de sementes. In: AGUIAR, I.B.; PIÑA-RODRIGUES, F.C.M.; FIGLIOLIA, M.B. (Coord.). Sementes florestais tropicais. Brasília: ABRATES, 1993. p. 83-135.

BRASIL. Ministério da Agricultura e da Reforma Agrária. Regras para análise de sementes. Brasília:SNDA/DNDV/CLAV, 1992. 365p.

CÍCERO, S.M. Dormência de sementes. In: SEMANA DE ATUALIZAÇÃO EM PRODUÇÃO DE SEMENTES. Piracicaba: FEALQ, 1986. p.14-74.

COPELAND, L.O.; MCDONALD, M.B. Principle of seed science and technology. New York: Chapmen \& Hall, 1995. 409p.

FIGLIOLIA, M.B.; OLIVEIRA, E.C.; PIÑARODRIGUES, F.C.M. Análise de sementes. In: AGUIAR, I.B.; PIÑA-RODRIGUES, F.C.M.; FIGLIOLIA, M.B 
(Coord.). Sementes florestais tropicais. Brasília: ABRATES, 1993. p.137-174.

FOWLER, J.A.P.; CARPANEZZI, A.A. Tecnologia de sementes de marica (Mimosa bimucronata (DC) O. Ktze.). Boletim de Pesquisa Florestal, Curitiba, n.36, p.47-56, 1998.

GOMES, S.M.S.; BRUNO, L. A. Influência da temperatura e substrato na germinação de sementes de urucum (Bixa orellana L.) Revista Brasileira de Sementes, Brasília, v.14, n.1, p. 47-50, 1992.

LABOURIAU, L.G. A germinação de sementes. Washington: OEA, 1983. 174p.

LABOURIAU, L.G.; AGUDO, M. On the physiology of seeds germination in Salvia hispanica L. I. Temperature effects. Anais da Academia Brasileira de Ciências, São Paulo, v.59, n.1, p.37-56, 1987.

LORENZI, H. Cnidosculus phyllacanthus (M. Arg.) Pax \& K. Hoffm. In: Árvores brasileiras: manual de identificação e cultivo de plantas arbóreas nativas do Brasil. Nova Odessa: Instituto Plantarum de Estudos da Flora, 1998. v.2, p.92.

LIMA, C.M.R., BORGHETTI, F.; SOUSA, M.V. Temperature and germination of the Leguminosae Enterolobium contortisiliquum. Revista Brasileira de Fisiologia Vegetal, Brasília, v.9, n.2, p.97-102, 1997.

MATOS, V.P.; SILVA, L.M.M.; LIMA. A.A. Efeito da temperatura e do substrato na germinação de sementes de mulungu (Erythrina velutina Willd.). In: CONGRESSO FLORESTAL PANAMERICANO, 1., CONGRESSO FLORESTAL BRASILEIRO, 7, 1993, Curitiba. Anais... Curitiba: SBS, 1993. v.2, p.758.

MAYER, A.C.; POLJAKOFF-MAYBER, A. The germination of seeds. London: Pergaman Press, 1989. $270 \mathrm{p}$.

MEDEIROS, A.C.; ZANON, A. Efeito do substrato e temperatura na germinação de sementes de branquilho (Sebastiania commersoniana). Boletim de Pesquisa Florestal, Curitiba, n.36, p.21-28, 1998.

MORENO-CASASOLA, P.; GRIME, J.P.; MARTÍNEZ, M.L. A comparative study of the flutuations in temperature
And moisture supply on hard coat dormancy in seeds of coastal tropical legumes in México. Journal of Tropical Ecology, v.10, p. 67-86, 1994.

OLIVEIRA, E.C.; PIÑA-RODRIGUES, F.C.M.; FIGLIOLIA, M.B. Propostas para padronização de metodologias em análise florestais. Revista Brasileira de Sementes, Brasília, v.11, n.123, p. 1-42, 1989.

PEREIRA, T.S.; ANDRADE, C.S. Germinação de Psidium guajava L. e Passiflora edulis Sims- Efeito da temperatura, substrato e morfologia do desenvolvimento pós-seminal. Revista Brasileira de Sementes, Brasília, v.16, n.1, p.5862, 1994.

POPINIGIS, F. Fisiologia da semente. Brasília: AGIPLAN, 1985. 285p.

SALOMÃO, A.N.; EIRA, M.T.S.; CUNHA, R. The effect of temperature on seed germination of four Dalbergia nigra Fr. Allem - Leguminosae. Revista Árvore, Viçosa, v.9, n.4, p.588-594, 1995.

SANTOS, S.R.G.; AGUIAR, I.B. Germinação de sementes de branquilho (Sebastiania commersoniana (Baill) Smith \& Down) em função do substrato e do regime de temperatura. Revista Brasileira de Sementes, Brasília, v.22, n.1, p.120$126,2000$.

SILVA, L.M.M.; AGUIAR, I.B.; DONADIO, N.M.M. Tratamento pré-germinativo em sementes de faveleira (Cnidosculus phyllacanthus Pax \& K. Hoffm.).In: CONGRESSO BRASILEIRO DE SEMENTES, 11., 1999, Foz de Iguaçu. Informativo ABRATES, Curitiba, 1999, v.9, n.1/2, p. 160 .

THOMPSON, K.; GRIME, J.P.; MASON, G. Seed germination in response to diurnal fluctuation of temperature. Nature, London, v.267, p.147-149, 1977.

VÁZQUEZ-YANES, C.; OROZCO-SEGOVIA, A. Seed germination of a tropical rain forest pioneer tree (Heliocarpus donnell-smithii) in response to diurnal fuctuation of temperature. Plant Physiology, New York, v.56, p.295-298, 1982.

VÁZQUEZ-YANES, C.; OROZCO-SEGOVIA, A. Fisiología ecológica de las semillas de árboles de la selva tropical. Ciência, v.35, p. 191-201, 1984. 